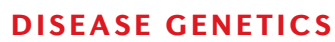

\section{Mitochondrial variation affects disease risk}

Genomic studies have identified thousands of variants associated with complex diseases. However, these variants can only provide an explanation for a portion of heritability (that is, the fraction of phenotypic variability in a population that can be attributed to genetic factors). Now, Hudson et al. show that mitochondrial variants affect the disease risk, which suggests that variation in the mitochondrial genome accounts for some of the missing heritability of complex diseases.

Mitochondrial DNA ( $m t D N A)$ encodes a finite number of proteins that affect the same energy production pathway, the disruption of which has been implicated in several diseases. Hence, the authors hypothesized that a given genetic variant of mtDNA could affect the risk of developing several different diseases. The authors computationally analysed common inherited mtDNA variants from $\sim 40,000$ affected individuals and $\sim 18,000$ healthy controls, and examined their effect on 11 different late-onset diseases such as multiple sclerosis. As predicted, they found several variants that altered the risk of developing more than one disease. Although some variants had protective effects, most variants increased the risk of disease. The authors propose that this bias towards deleterious variants may be because these mtDNA alleles escape purifying selection, as they affect diseases that are apparent only after the end of female reproductive life.

Although preliminary, these findings highlight the possible contribution of mtDNA variance to the risk of complex diseases and suggest that the mitochondrial genome should be included in large-scale genetic association studies.

Isabel Lokody

ORIGINAL RESEARCH PAPER Hudson, G. et al. Recent mitochondrial DNA mutations increase the risk of developing common late-onset human diseases. PLoS Genet. 10, e1004369 (2014)

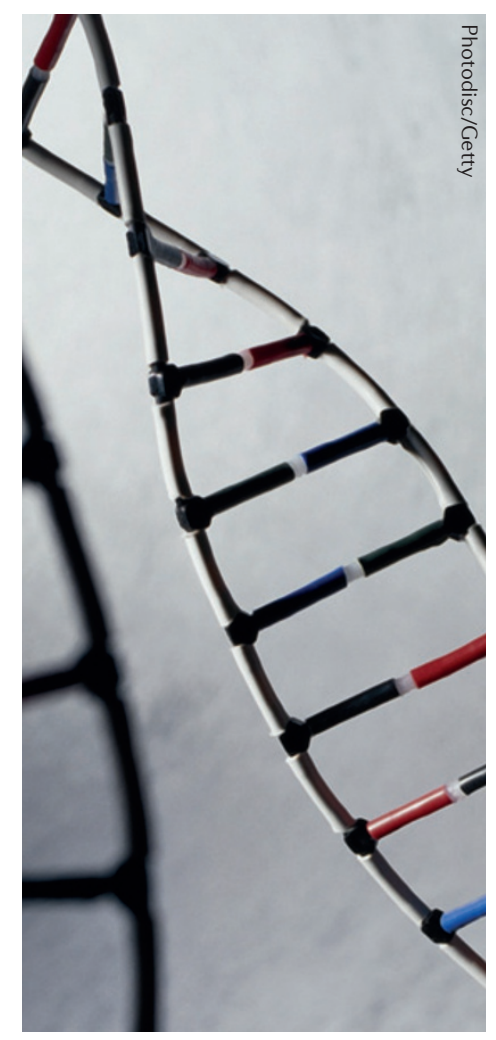

